\title{
ON THE NATURE OF THE BINARY COMPONENTS OF RX J0806.3+1527
}

\author{
K. Rcinsch, ${ }^{1,3}$ V. Burwitz, ${ }^{2}$ and R. Schwarz ${ }^{1}$ \\ RESUMEN
}

\begin{abstract}
Presentamos polarimetría circular y fotometría en el infrarrojo cercano de RX J0806.3+1527, que se sospecha es una binaria enana blanca con un periodo ultra-corto, obtenida con VLT ESO y discutimos las implicaciones de una posible natıraleza magnética de la enana blanca acreciente y las correspondientes restricciones derivadas de ello para la estrella donante. Nuestros datos en el filtro $V$ muestran, una polarización circular marginalmente significativa con una modulación en la amplitud de $\approx 0.5 \%$, típico de una emisión ciclotron procedente de una columna de acreción en un campo magnético del orden de $10 \mathrm{MG}$ e incompatible con un modelo de impacto directo. La distribución de flujo desde el óptico al infrarrojo cercano está bien descrita por la emisión de un único cuerpo negro con una temperatura $k T_{\mathrm{bb}}=35000 \mathrm{~K}$ y excluyc la posibilidad de que el donante sea una estrella en la secuencia principal a no ser que la binaria esté localizada a varias alturas de escala sobre la población del disco galíctico.
\end{abstract}

\section{ABSTRACT}

We present imaging circular polarimetry and near-infrared photometry of the suspected ultra-short period white-dwarf binary RX J0806.3+1527 obtained with the ESO VLI and discuss the implications for a possible nuagnetic nature of the white dwarf accretor and the constraints derived for the nature of the donor star. Our $V$-filter data show marginally significant circular polarization with a modulation amplitude of $\approx 0.5 \%$ typical for cyclotron emission from an accretion column in a magnetic field of order $10 \mathrm{MG}$ and not compatible with a direct-impact accretor model. The optical to near-infrared flux distribution is well described by a single blackbody with temperature $k T_{\mathrm{bb}}=35000 \mathrm{~K}$ and excludes a main-sequence stellar donor unless the binary is located several scale heights above the galactic disk population.

\section{Key Words: BINARIES: CLOSE - POLARIZATION — STARS: INDIVIDUAL (RX J0806.3+1527) - STARS: LATE-TYPE - STARS: MAGNETIC FIELDS}

\section{INTRODUCTION}

The soft X-ray discovered system RX J0806.3 +1527 (Beuermann of al. 1999) has recently been suggested to be a semidetached white dwarf binary with a helium-degenerate secondary and the shortest known orbital period Israel et al. (2002). If the $321 \mathrm{~s}$ pulse period (Israel et al. 1999. Burwitz \& Reinsch 2001 ) is indeed the orbital period this system would lie close to the theoretical minimum period of white $\mathrm{d}$ warf binaries and would be a suitable candidate for gravitational wave detection.

Three flavors of the double-degenerate model (polar, direct accretor, electric star) have been advanced to account for the observational characteristics (Cropper et al. 1998, Marsh \& Steeghs 2002, Wu et al. 2002). Alternatively, an interpretation of RX.J0806 as a face-on, stream-fed intermediate polar has been advocated (Norton et al. 2003).

\footnotetext{
${ }^{1}$ Universitäts-Sternwarte, Göttingen, Germany.

2 MPI f. extraterr. Physik, Garching, Germany.

${ }^{3}$ Based on observations collected at the European Southern Observatory, Chile under proposal number 70.D-0683.
}

We present the results of our recent imaging circular polarimetry and near-infrared photometry and discuss the implications for a possible magnetic nature of the white dwarf accretor and the constraints derived for the nature of the donor star.

\section{CIRCULAR POLARIMETRY}

Time-resolved circular polarimetry of RX J0806 has been obtained with FORS1 at the ESO VLT during 7 nights between December 2002 and February 2003. Our data cover a total of $3 \mathrm{~h}$ through a Bessel $V$ filter and $3 \mathrm{~h}$ in Bessel $I$ at $\approx 60 \mathrm{~s}$ time resolution corresponding to a phase resolution $\Delta \Phi \approx 0.2$ of the 321 s period.

Stokes $V$ and $I$ data have been derived from the Bessel $V$ and $I$ images using aperture photometry (Fig. 1). The $321 \mathrm{~s}$ period phase-folded data show a marginally significant circular polarization with a modulation amplitude of $\approx 0.5 \%$ in Bessel $V$. The signal-to-noise of the $I$ filter data is much lower allowing us to derive only an upper limit for the $I$-band circular polarization $V_{\text {mean }} \lesssim 2 \%$. 


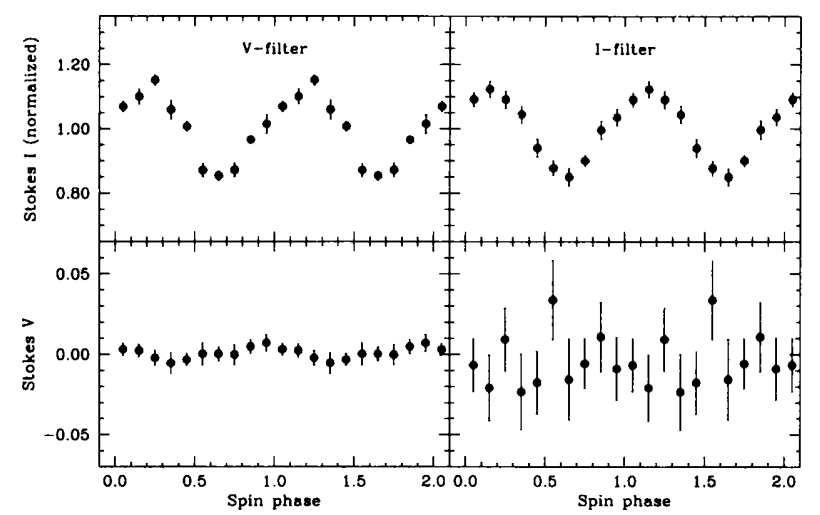

Fig. 1. Normalized intensity variation (upper panels) and circular polarization (lower panels) of RX J0806.3+1527 observed in the Bessel $V$ (left side) and Bessel I (right side) filters, respectively. All data have been phase folded with $P=321.5393 \mathrm{~s}$ and arbitrary zero point.

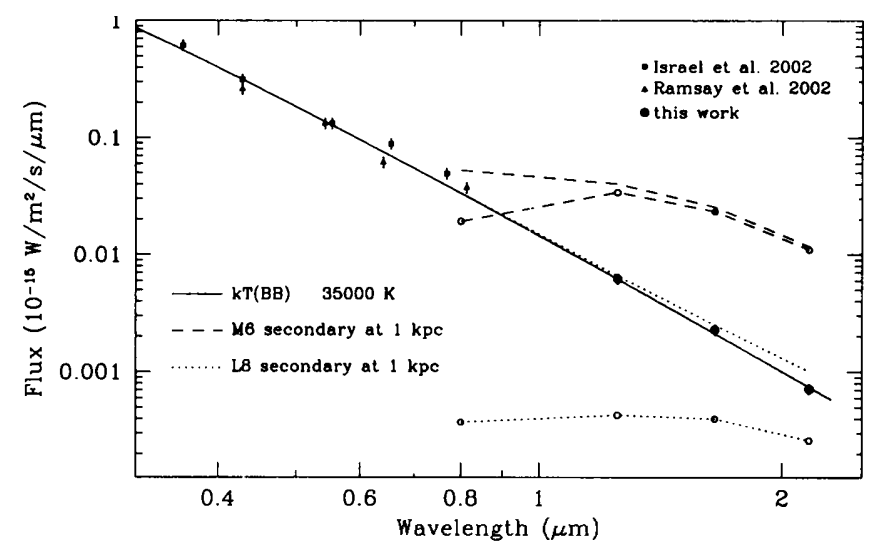

Fig. 2. Optical and near-infrared flux distribution of $\mathrm{RX} \mathrm{J} 0806.3+1527$. The straight line represents a $k T_{\mathrm{bb}}=$ $35000 \mathrm{~K}$ blackbody. For comparison the flux contributions and total fluxes expected for a late-type secondary at $1 \mathrm{kpc}$ distance are shown (dashed line: M6 star, dotted line: L8 star).

\section{INFRARED PHOTOMETRY}

Two hours of time-resolved jittered infrared imaging of RX J0806 in each of the $J, H$, and $K s$ filter bands has been performed with ISAAC SW at the ESO VLT under photometric conditions during 5 nights between January and March 2003. Effective integration times were $60 \mathrm{~s}$ per image.

The average fluxes of RX J0806 in the $J, H$, and $K s$ filter bands significantly extend the optical flux distribution obtained by Israel et al. (2002) and Ramsay et al. (2002) (Fig. 2). The optical to nearinfrared flux distribution is well described by a single blackbody with temperature $k T_{\mathrm{bb}}=35000 \mathrm{~K}$.

\section{DISCUSSION}

The circular polarization detected in the $V$-filter data is comparable to that seen in some intermediate polars (e.g. Buckley et al. 1995). This is char- acteristic of cyclotron emission originating from an accretion column in a magnetic field of order $10 \mathrm{IG}$ and would be compatible with all currently discussed models except for the direct accretor.

The optical-to-infrared flux distribution excludes a main-sequence stellar donor unless the binary is located several scale heights above the galactic disk population. If the system is at a distance of $1 \mathrm{kpc}$ (i.e. at approximately twice the galiact ic scale height) the secondary must be of spectral type L 8 or later that its flux contribution can be hidden in the observed spectrum. This is difficult to conceal in an intermediate polar interpretation of RX J0806 which implies a Roche-lobe filling stellar companion.

On the other hand, the question whether other periods are present in the system (as expected for an intermediate polar) is not well settled and needs further observations. Furthermore, the evidence for Hydrogen in the optical spectrum of RX J0806 implies severe problems for all ultra-short period binary models which require a Helium rich or a Helium degenerate secondary.

Concluding, none of the models proposed so far fits well with all available observations and the true nature of RX J0806.3+1527 must still be considered open.

We thank ESO staff nombers at Paranal observatory for obtaining the clata as part of an ESO Service Mode run. This project has been supported in part by BMBF/DLR grant 50 OR 0206 .

\section{REFERFNCES}

Beuermann, K.. Thomas, H. C.. Reinsch, K.. Schwope. A. D., Trümper, J., \& Voges, II. 1999, AL 1. 347. 47 Buckley, D. A. H., Sekiguchi, K., Motch, et al. 1995. M. RAS, 275, 1028

Burwitz, V., \& Reinsch, K. 2001, AIP Conf. Proc.. 599. 522

Cropper, M., Harrop-Allin. M. K.. Mason, K. O.. Mittaz. J. P. D., Potter, S. B., Ramsay: G. 1998, MNRAs. 293, L57

Israel, G. L., Panzera, M. R., Campana. S., Lazaati. D. Covino, S., Tagliaferri, G., \& Stella, L. 1999. 1\$. $349, \mathrm{~L} 1$

Israel, G. L., Hummel, W., Covino, S., et al. 2002. At.A. 386, L13

Marsh, T. R., \& Steeghs, D. 2002, MNRAS. 331. Li

Norton, A. J., Haswell, C. A.. \& WYmu, G. A. 2003, MNRAS, in press, astro-ph/0206013

Ramsay, G., Hakalia. P.. \& Cropper, .I. 2002. MNRAS. 332, L7

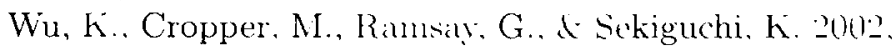
MNRAS. 331, 221 